\title{
Expression of p-Akt in ovarian serous carcinoma and its association with proliferation and apoptosis
}

\author{
TIEYING SONG ${ }^{1 *}$, LIWEN WANG $^{2 *}$, ZHONGFU MO $^{2}$, LIMEI MAO $^{2}$, XIAOJING MA ${ }^{1}$, RUNLING NIU $^{1}$, \\ KUNFENG GU ${ }^{1}$, RUYU YAN ${ }^{1}$, PENGYU MA ${ }^{1}$, YAN QI ${ }^{1}$ and QINGFANG JIAO ${ }^{3}$ \\ ${ }^{1}$ Department of Anesthesiology, The First Hospital of Shijiazhuang, Shijiazhuang, Hebei 050011; \\ ${ }^{2}$ Department of Gynecology, The Sixth Hospital of Shijiazhuang, Shijiazhuang, Hebei 050000; \\ ${ }^{3}$ Department of Neurosurgery, The First Hospital of Shijiazhuang, Shijiazhuang, Hebei 050011, P.R. China
}

Received April 3, 2013; Accepted October 17, 2013

DOI: $10.3892 / \mathrm{ol} .2013 .1641$

\begin{abstract}
The aim of the present study was to determine the expression of p-Akt in ovarian serous carcinoma (OSC) and its association with proliferation and apoptosis. Paraffin-embedded tissues of patients aged between 35 and 64 years old without history of radiotherapy, chemotherapy and hormone therapy prior to surgery were collected. In total, samples included 12 ovarian serous cystadenomas (OSAs), 18 ovarian serous borderline tumors (OS-BTs) and 46 OSCs. Of the 46 OSC samples, 16 were well-differentiated, 20 were moderately differentiated and 10 were poorly differentiated, while 22 developed lymphatic metastases and 24 were metastasis-free. An additional 10 paraffin-embedded normal ovarian tissues (NOTs) were used as controls. Streptavidin-peroxidase immunohistochemistry assays were used to investigate the expression of p-Akt and cyclin D1 in the collected samples. Compared with NOT, p-Akt expression in the OS-BT and OSC groups, as well as cyclin D1 expression in the OSA and OSC groups, was significantly elevated $(\mathrm{P}<0.05)$. Compared with the OSA group, p-Akt expression in the OSC group was significantly elevated $(\mathrm{P}<0.01)$ and reversely associated with tumor differentiation $(\mathrm{P}<0.01)$, whereas cyclin $\mathrm{D} 1$ expression showed no correlation with tumor differentiation $(\mathrm{P}>0.05)$. The expression of $\mathrm{p}-\mathrm{Akt}$, caspase- 3 and cyclin D1 was positively associated with lymphatic metastasis $(r=0.334$; $\mathrm{P}=0.023)$. The expression of $\mathrm{p}-\mathrm{Akt}$ gradually increased with carcinoma development and was associated with differentiation and metastasis of OSC, revealing that the activation of the PI3K/Akt signaling pathway is involved in the development
\end{abstract}

Correspondence to: Professor Tieying Song, Department of Anesthesiology, The First Hospital of Shijiazhuang, 36 Fanxi Road, Shijiazhuang, Hebei 050011, P.R. China

E-mail: songtieyingBM@163.com

*Contributed equally

Key words: phosphatidylinositol-3-kinase/protein kinase B, cyclin D1, proliferation, apoptosis, ovarian serous carcinoma of OSC. Furthermore, the expression of cyclin D1 gradually increased in the NOT, OSA, OS-BT and OSC groups and was associated with tumor metastasis.

\section{Introduction}

Ovarian carcinomas account for only $15-20 \%$ of female malignant carcinomas in the US; however, these tumors have the highest mortality rate. Ovarian serous carcinoma (OSC) accounts for $40 \%$ of all ovarian carcinomas in the US and is the leading cause of morbidity and mortality in malignant carcinoma of the female reproductive system (1). Despite applied chemotherapies and cytoreductive surgery combined therapies, the five-year survival rate of OSC is only $30-40 \%$ (2). Recent studies have found that ovarian carcinoma is a disease that is characterized by simultaneous excessive cell proliferation and decreased apoptosis, with uncontrolled proliferation and blocked apoptosis playing a crucial role in tumorigenesis $(3,4)$. It is important to study the mechanisms and genes associated with proliferation and apoptosis in ovarian carcinoma, particularly for OSC, which may highlight new gene-therapeutic methods that simultaneously inhibit proliferation and induce apoptosis of cancer cells.

The phosphatidylinositol-3-kinase/protein kinase B (PI3K/Akt) signaling pathway was identified in 1987 by Staal who found an oncogene known as serine/threonine-specific protein kinase (Akt) in the murine retrovirus AKT8 after producing foci of malignant transformation in the mink lung epithelial cell line, CCL 64 (5). Akt is a 60-kDa serine/threonine-specific protein kinase prevalent in eukaryotic regulatory networks and has an important junction point which links multiple signal transduction pathways, regulates multiple extracellular cytokine signal transductions and is important for Ras-mediated oncogenic transformation (6). The PI3K/Akt signaling pathway is also involved in cell metabolism, regulation of the cell cycle and angiogenesis, and is associated with the development of diabetes and multiple autoimmune diseases, including rheumatoid arthritis. Deficiency or inactivation of the PI3K/Akt signaling pathway-associated regulatory genes, mutation or amplification of the PI3K gene and activation of receptors or junction molecules of its downstream signaling pathway have been identified in multiple tumor cell lines (7). 
Cyclin-dependent kinases (CDKs) are crucial for the cell division cycle $(8,9)$. The cell cycle is coregulated by CDKs and cyclin-dependent kinase inhibitors (CDKIs), as well as cyclin D1 levels, which are essential for the G1 to S phase cell cycle transition. However, little is known with regard to the association of Akt with the cell cycle progression.

Based on a previous study (10), pathomorphology and immunohistochemistry were used in the current study to investigate the expression of p-Akt and cyclin D1 in normal ovarian tissue (NOT), ovarian serous cystadenoma (OSA), ovarian serous borderline tumors (OS-BT) and OSC samples to further investigate the role of p-Akt in the development of ovarian epithelial cancer and its possible effect on cyclin D1 signaling pathway activation.

\section{Materials and methods}

Tissue samples. Paraffin-embedded tissues were collected from the First Hospital of Shijiazhuang (Shijiazhuang, China). All tissues originated from patients aged between 35 and 64 years old without history of radiotherapy, chemotherapy and hormone therapy prior to surgery. Among the collected samples, 12 were OSA, 18 were OS-BT and 46 were OSC. Of the 46 OSC samples, 16 were well-differentiated, 20 were moderately differentiated and 10 were poorly differentiated, while 22 OSC samples led to lymphatic metastasis and 24 were metastasis-free. An additional 10 paraffin-embedded NOTs were used as control. The current study was approved by the ethics committee of the First Hospital of Shijiazhuang and the Sixth Hospital of Shijiazhuang. Informed written consent was obtained from all participants.

Immunohistochemistry. Immunohistochemistry S-P assays were used to investigate the expression of p-Akt in NOT, OSA, OS-BT and OSC samples. In total, 10 high power fields were randomly selected from each paraffin section and examined under a light microscope (Olympus, Tokyo, Japan) using the double-blind method. All slides were processed with polylysine prior to immunohistochemical staining for p-Akt and cyclin D1 protein visualization. Rabbit anti-human p-Akt monoclonal antibodies (Cell Signaling Technology, Inc., Danvers, MA, USA) and rabbit anti-human cyclin D1 monoclonal antibodies (ready-to-use; Fuzhou Maixin Biotechnology Development Co., Ltd., Fuzhou, China) were used as primary antibodies, and goat anti-rabbit IgG/biotin (Fuzhou Maixin Biotechnology Development Co., Ltd.) was used as the secondary antibody. Phosphate-buffered saline (PBS; $0.01 \mathrm{~mol} / \mathrm{l}$ ) only was used as a control for primary antibodies.

Sample preparation. Paraffin-embedded samples were prepared into $4-\mu \mathrm{m}$ sections and deparaffinized by a standard method. The paraffin sections were then submerged in hydrogen peroxide methanol solutions and vortexed at room temperature for 15 min to block the bioactivity of endogenous peroxidases. Next, sections were rinsed twice for 5 min with distilled water and placed into plastic boxes filled with antigen retrieval buffer $(0.01 \mathrm{~mol} / \mathrm{l}$ citric acid/sodium citrate solution, pH 6.0; Fuzhou Maixin Biotechnology Development Co., Ltd.) for initial microwave treatment at 7th gear for 5 min followed by a second treatment at 4 th gear for $3 \mathrm{~min}$. The boxes were then

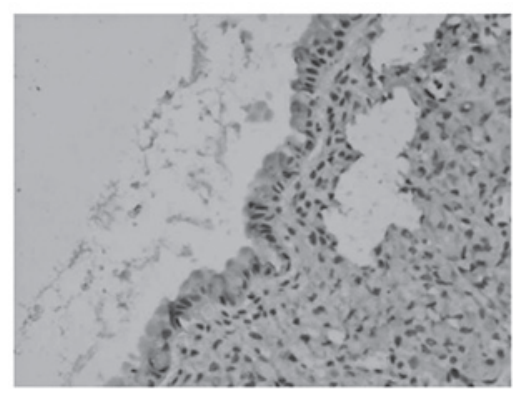

Figure 1. Negative expression of p-AKT in NOT (magnification, x100). NOT, normal ovarian tissue.

cooled at room temperature for 15-20 min. Next, sections were rinsed three times for $5 \mathrm{~min}$ with $0.01 \mathrm{~mol} / \mathrm{l} \mathrm{PBS}$ and normal goat serum was added at $37^{\circ} \mathrm{C}$ for $30 \mathrm{~min}$ to block endogenous biotin. Serum was discarded and primary antibodies (rabbit anti-human p-Akt monoclonal antibody, 1:200; and rabbit anti-human cyclin D1 monoclonal antibody, ready-to-use) were added separately for overnight incubation in a humid atmosphere at $4^{\circ} \mathrm{C}$. Sections were then rinsed three times for 5 min with $0.01 \mathrm{~mol} / 1 \mathrm{PBS}$, and secondary antibody (goat anti-rabbit $\mathrm{IgG} /$ biotin) was added for $25 \mathrm{~min}$ at $37^{\circ} \mathrm{C}$, followed rinsing three times for $5 \mathrm{~min}$ with $0.01 \mathrm{~mol} / \mathrm{l} \mathrm{PBS}$. Next, streptavidin/horseradish peroxidase was added for $20 \mathrm{~min}$ at $37^{\circ} \mathrm{C}$ and the sections were rinsed four times for $5 \mathrm{~min}$ with $0.01 \mathrm{~mol} / \mathrm{l}$ PBS. Finally, freshly prepared DAB- $\mathrm{H}_{2} \mathrm{O}_{2}$ (Fuzhou Maixin Biotechnology Development Co., Ltd.) was added for color development, which was monitored under a light microscope (Olympus CX21; Olympus Corporation, Tokyo, Japan) and rinsed again with distilled water to terminate the reaction. Hematoxylin (BASO Precision Optics Ltd., Taiching, Taiwan) was added for slight re-staining. Sections were differentiated with hydrochloric acid alcohol, dehydrated with an ascending series of ethanol, cleared in xylene and mounted in neutral balsam. Positive samples and negative controls were set up during the process. The procedure was used for all sample sections.

Immunochemistry analysis. Selecting a homogeneously stained positive region and grading the proportion of stained cells compared with all cells within the field of vision was scored as follows: 0 , no positively stained cells; $1,<25 \%$ positively stained cells; $2,25-50 \%$ positively stained cells; and $3,>50 \%$ positively stained cells. Grading was determined by the color intensity of stained cells and was as follows: 0 , negative; 1, weak light yellow; 2, medium brown yellow; and 3, strong dark brown. Results were analyzed by adding the above values together and were determined as follows: 0-2, negative (-); 3-4, weak-positive (+); and 5-6, strong-positive (++). Weak-positive (+) and strong-positive (++) were considered as positive.

Statistical analysis. Data were analyzed using SPSS version 11.0 (SPSS, Inc., Chicago, IL, USA) and quantitative data are presented as the medium \pm standard deviation. Values between groups were compared by one-way analysis of variance and counting data were analyzed by the $\chi^{2}$ test. $\mathrm{P}<0.05$ was considered to indicate a statistically significant difference. 
Table I. Expression of p-Akt in different groups.

\begin{tabular}{lcccc}
\hline & & \multicolumn{2}{c}{$\mathrm{p}$-Akt expression, $\mathrm{n}(\%)$} & \\
\cline { 3 - 4 } Groups & $\mathrm{n}$ & - & + & P-value \\
\hline NOT & 10 & $10 / 10(100.00)$ & $0 / 10(0.00)$ & \\
OSA & 12 & $10 / 12(83.33)$ & $2 / 12(16.67)$ & 0.481 \\
OS-BT & 18 & $8 / 18(44.44)$ & $10 / 18(55.56)$ & 0.004 \\
OSC & 46 & $16 / 46(34.78)$ & $30 / 46(65.22)$ & 0.000
\end{tabular}

NOT, normal ovarian tissue; OSA, ovarian serous cystadenoma; OS-BT, ovarian serous borderline tumor; OSC, ovarian serous carcinoma.

Table II. Expression of p-Akt in different pathological grades of OSC.

\begin{tabular}{lllll}
\hline & & \multicolumn{2}{c}{$\mathrm{p}$-Akt expression, $\mathrm{n}(\%)$} & \\
\cline { 3 - 4 } Pathological grade & $\mathrm{n}$ & \multicolumn{1}{c}{$\begin{array}{l}\text { P } \\
\text { P-value }\end{array}$} \\
\hline G1 & 16 & $9 / 16(56.25)$ & $7 / 16(43.75)$ & \\
G2 & 20 & $7 / 20(35.00)$ & $13 / 20(65.00)$ & 0.014 \\
G3 & 10 & $0 / 10(0.00)$ & $10 / 10(100.00)$ &
\end{tabular}

OSC, ovarian serous carcinoma.

Table III. Correlation between the expression of p-Akt and infiltration and metastasis of cancer tissue.

\begin{tabular}{lrr}
\hline & \multicolumn{2}{c}{$\mathrm{p}$-Akt expression } \\
\cline { 2 - 3 } Infiltration or metastasis & \multicolumn{1}{c}{+} \\
\hline- & 12 & 12 \\
+ & 4 & 18 \\
\hline $\mathrm{r}=0.334 ; \mathrm{P}=0.023$. & & \\
\hline
\end{tabular}

\section{Results}

Expression of $p$-Akt in ovarian carcinoma. p-Akt was predominantly located in the nuclei and cytoplasm of ovarian carcinoma cells, appearing as dark brown sediments (Figs. 1 and 2). Based on the immunohistochemistry S-P assay results (Tables I-III), p-Akt prevalence was significantly different between the NOT, OSA, OS-BT and OSC groups $\left(\chi^{2}=19.781 ; \mathrm{P}<0.01\right)$. In the OSC samples, the prevalence of $\mathrm{p}$-Akt expression was reversely associated with tumor differentiation $(\mathrm{P}<0.01)$. p-Akt prevalence was positively associated with lymphatic metastasis $(\mathrm{r}=0.334$; $\mathrm{P}=0.023$ ) and a higher $\mathrm{p}$-Akt prevalence was observed in OSC samples with lymphatic metastasis compared with metastasis-free OSC samples $(\mathrm{P}<0.05)$.
Table IV. Expression of cyclin D1 in different groups.

\begin{tabular}{lccrr}
\hline & & \multicolumn{2}{c}{ Cyclin D1 expression, $\mathrm{n}(\%)$} & \\
\cline { 3 - 4 } Group & $\mathrm{n}$ & - & + & P-value \\
\hline NOT & 10 & $9 / 10(100.00)$ & $1 / 10(10.00)$ & \\
OSA & 12 & $9 / 12(75.00)$ & $3 / 12(25.00)$ & 0.594 \\
OS-BT & 18 & $8 / 18(44.44)$ & $10 / 18(55.56)$ & 0.041 \\
OSC & 46 & $12 / 46(26.09)$ & $34 / 46(73.91)$ & 0.000 \\
\hline
\end{tabular}

NOT, normal ovarian tissue; OSA, ovarian serous cystadenoma; OS-BT, ovarian serous borderline tumor; OSC, ovarian serous carcinoma.

Table V. Expression of cyclin D1 in different pathological grades of OSC.

\begin{tabular}{|c|c|c|c|c|}
\hline \multirow[b]{2}{*}{ Pathological grade } & \multirow[b]{2}{*}{$\mathrm{n}$} & \multicolumn{2}{|c|}{$\begin{array}{c}\text { Cyclin D1 expression, } \\
\mathrm{n}(\%)\end{array}$} & \multirow[b]{2}{*}{ P-value } \\
\hline & & - & + & \\
\hline G1 & 16 & $5 / 16(31.25)$ & $11 / 16(68.75)$ & \\
\hline G2 & 20 & $6 / 20(30.00)$ & $14 / 20(70.00)$ & 0.161 \\
\hline G3 & 10 & $1 / 10(10.00)$ & 9/10 (90.00) & \\
\hline
\end{tabular}

OSC, ovarian serous carcinoma.

Table VI. Correlation between the expression of cyclin D1 and the infiltration and metastasis of OSC.

\begin{tabular}{lrr}
\hline & \multicolumn{2}{c}{ Cyclin D1 expression } \\
\cline { 2 - 3 } Infiltration or metastasis & \multicolumn{1}{c}{} \\
\hline- & 10 & + \\
+ & 2 & 14 \\
\hline
\end{tabular}

$\mathrm{r}=0.371 ; \mathrm{P}=0.011$. OSC, ovarian serous carcinoma.

Expression of cyclin D1 in ovarian carcinoma. Cyclin D1 was predominantly located in the nuclei and cytoplasm of ovarian carcinoma cells, appearing as brown nuclei and dark brown sediments in the cytoplasm (Fig. 3). Based on the immunohistochemistry S-P assay results (Tables IV-VI), the prevalence of cyclin D1 expression was significantly different among the NOT, OSA, OS-BT and OSC groups $\left(\chi^{2}=19.241\right.$; $\mathrm{P}<0.01)$. OSA and OSC groups exhibited significantly higher cyclin D1 levels compared with the NOT group $(\mathrm{P}<0.05$ and $\mathrm{P}<0.01$, respectively). No significant difference in the prevalence of cyclin D1 expression was observed among the three tumor differentiation stages within the 46 OSC samples $(\mathrm{P}>0.05)$. The prevalence of cyclin D1 expression was positively associated with lymphatic metastasis $(r=0.371$; $\mathrm{P}=0.011$ ), since a higher cyclin D1 prevalence was observed 


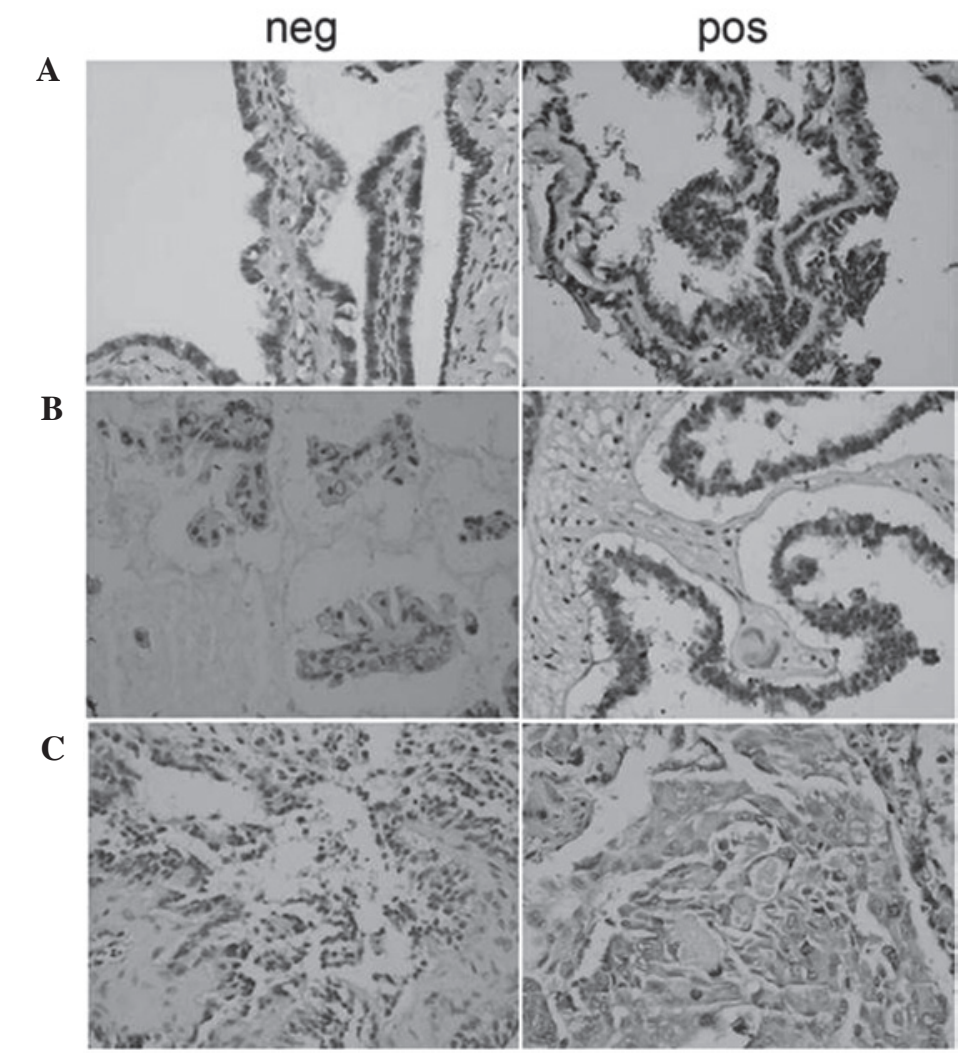

Figure 2. p-Akt expression patterns in (A) OSA, (B) OS-BT and (C) OSC. Left and right images represent negative and positive immunohistochemical staining, respectively (magnification, x100). OSA, ovarian serous cystadenoma; OS-BT, ovarian serous borderline tumor; OSC, ovarian serous carcinoma.
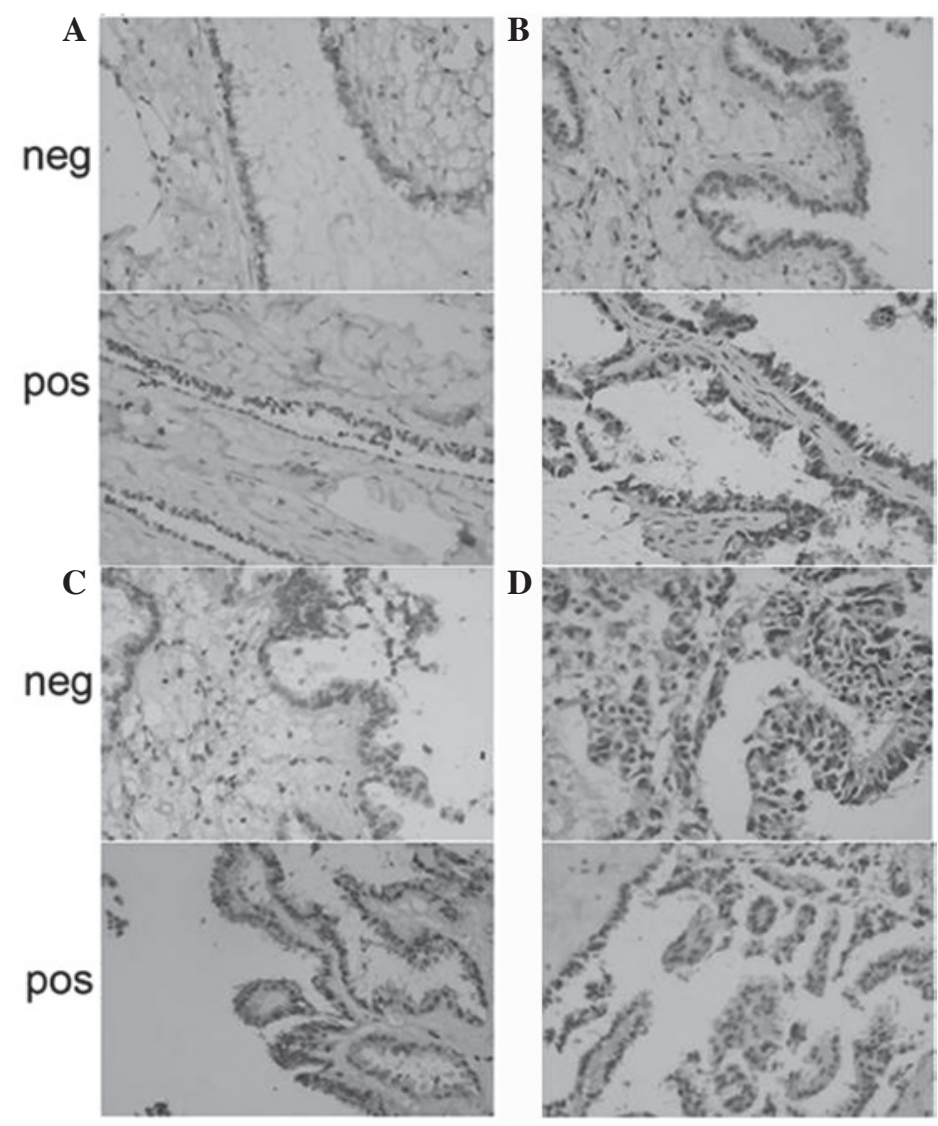

Figure 3. Cyclin D1 expression patterns in (A) NOT, (B) OSA (C) OS-BT and (D) OSC. Upper and lower panels represent negative and positive immunohistochemical staining, respectively (magnification, x100). NOT, normal ovarian tissue; OSA, ovarian serous cystadenoma; OS-BT, ovarian serous borderline tumor; OSC, ovarian serous carcinoma. 
in OSC samples with lymphatic metastasis compared with metastasis-free OSC samples $(\mathrm{P}<0.05)$.

Association of $p$-AKT, caspase-3 and cyclin D1. The prevalence of p-Akt expression was positively associated with the prevalence of cyclin D1 expression $(\mathrm{P}<0.001)$, but negatively associated with the prevalence of caspase-3 expression $(\mathrm{P}=0.017)$ (Tables VII and VIII).

\section{Discussion}

The Akt gene, also known as protein kinase B, is an oncogene that was identified in 1987 by Staal and recognized as a serine/threonine-specific protein kinase (5). Akt was cloned in 1991 and is composed of an N-terminal regulatory domain, central kinase domain and C-terminal regulatory domain, as well as a hinge region. Phosphorylation of Akt at Ser473 and Thr308 is essential for the activation of p-Akt. Activated Akt relocates to the cytoplasm and nucleus where it phosphorylates multiple substrates to activate or inhibit downstream targets, including Bad (a Bcl-2 family member) (11), nuclear factor $\kappa B$ (12), glycogen synthase kinase-3 (GSK-3) (13), transcription regulatory proteins and other proteins involved in the regulation of cell proliferation, differentiation and apoptosis. Expression levels of p-Akt indicate the bioactivity of the PI3K/Akt signaling pathway. In the current study, immunohistochemistry S-P assays were used to detect the expression of p-Akt in ovarian carcinoma tissues. No p-Akt expression was identified in NOTs, but positive expression rates of 16.7, 55.6 and $82.6 \%$ were identified in OSA, OS-BT and OSC tissues, respectively. The positive expression rates of p-Akt in well-, moderately and poorly differentiated ovarian carcinoma were 43.6, 65.0 and $80.0 \%$, respectively. The prevalence of p-Akt expression in ovarian carcinoma tissue with lymphatic metastasis was $81.8 \%$ and in metastasis-free ovarian carcinoma tissue was $50.0 \%$. Statistical analyses indicated that the prevalence of p-Akt expression was significantly different among the NOT, OSA, OS-BT and OSC groups $\left(\chi^{2}=19.781 ; \mathrm{P}<0.01\right)$, particularly, between the NOT and OS-BT, OSA and OS-BT and OSA and OSC groups $(\mathrm{P}<0.01)$. Among the 46 OSC samples, p-Akt occurrence was negatively associated with the degree of tumor differentiation $(\mathrm{P}<0.01)$ and statistical analysis also revealed that $\mathrm{p}$-Akt prevalence was positively associated with lymphatic metastasis $(r=0.334 ; \mathrm{P}=0.023)$, since OSC tissue with lymphatic metastasis exhibited significantly higher $\mathrm{p}-\mathrm{Akt}$ levels compared with metastasis-free OSC tissue. Previous studies have reported a possible involvement of the PI3K/Akt signaling pathway in ovarian carcinoma development and its clinical implications. Philp et al found that the p85 subunit of PI3K may be a new ovarian carcinoma oncogene and that mutations in PI3KCA may play critical roles in the development of ovarian carcinoma (14). Based on immunohistochemistry assay results, Noske et al found that Akt expression was $58 \%$ higher in primary ovarian carcinoma compared with that in NOTs, and it was significantly associated with positive lymph node rates and International Federation of Gynecology and Obstetrics stages (15). In addition, western blot analyses revealed that positive Akt expression in all investigated ovarian carcinoma cell lines and gonadal hormones increased the invasion and metastasis of epithelial ovarian carcinoma
Table VII. Correlation between the expression of p-Akt and cyclin D1 in OSC.

\begin{tabular}{lrr}
\hline & \multicolumn{2}{c}{ Cyclin D1 expression } \\
\cline { 2 - 3 } p-Akt expression & + & - \\
\hline+ & 28 & 2 \\
- & 6 & 10 \\
\hline
\end{tabular}

$\mathrm{r}=0.606 ; \mathrm{P}=0.000$. OSC, ovarian serous carcinoma.

Table VIII. Correlation between the expression of p-Akt and caspase-3 in OSC.

Caspase-3 expression

\begin{tabular}{lll}
\cline { 2 - 3 } p-Akt expression & + & - \\
\hline+ & 8 & 22 \\
- & 6 & 10 \\
\hline
\end{tabular}

$\mathrm{r}=-0.350 ; \mathrm{P}=0.017$. OSC, ovarian serous carcinoma.

cells via activation of the PI3K/Akt signaling pathway, which is consistent with the results of the current study.

Uncontrolled proliferation is a critical marker of malignant carcinoma, as vigorous proliferative activity of carcinoma cells is the basis and prerequisite of carcinoma invasion and metastasis. Proliferation of carcinoma cells is regulated by complex signaling pathways and dysfunctional cell cycle regulatory mechanisms. The cell cycle is coregulated by CDKs and CDKIs and a combination of cyclins with CDKs is essential for the activation of CDKs. Cyclin D1 is one of the most important cyclins, playing a crucial role in the transition in the G1/S cell cycle phase and controls the initiation of the cell cycle and mitosis completion $(16,17)$. In the present study, immunohistochemistry S-P assays were used to detect the expression of cyclin D1 in ovarian carcinoma tissues and found an increasing trend in the positive staining rates of NOT, OSA, OS-BT and OSC samples from $10.0,25.0,55.6$ to $73.9 \%$, respectively. The positive rates of cyclin D1 in well-, moderately- and poorly-differentiated ovarian carcinoma were 68.8, 70.0 and $90.0 \%$, respectively. Ovarian carcinoma tissue with lymphatic metastasis showed a $90.9 \%$ prevalence of cyclin D1 expression and a $50.0 \%$ prevalence in metastasis-free ovarian carcinoma tissue. Statistical analyses indicated that cyclin D1 expression was significantly different among the NOT, OSA, OS-BT and OSC groups $\left(\chi^{2}=19.241 ; \mathrm{P}<0.01\right)$. The prevalence of cyclin D1 expression in the OSA and OSC groups was significantly higher compared with that in the NOT group $(\mathrm{P}<0.05$ and $\mathrm{P}<0.01$, respectively). Among the 46 OSC samples, the prevalence of cyclin D1 expression did not significantly vary within the three tumor differentiation stages $(\mathrm{P}>0.05)$. An additional statistical analysis also revealed that cyclin D1 expression was positively associated with lymphatic metastasis $(r=0.371$; $\mathrm{P}=0.011$ ), while cyclin D1 prevalence in OSC samples with 
lymphatic metastasis was significantly higher compared with metastasis-free OSC samples $(\mathrm{P}<0.05)$. Lee et al reported that the positive rates of cyclin D1 expression were increased in the NOT, OSA, OS-BT and OSC groups $(\mathrm{P}<0.05)$ and correlated with tumor differentiation, clinical stages and lymphatic metastasis, which is consistent with the results of the current study (18).

In the present study, the expression levels of p-Akt and cyclin D1 were analyzed in OSC samples and the prevalence of p-Akt expression was found to positively correlate with that of cyclin D1, indicating an association between the PI3K/Akt signaling pathway and OSC proliferation and apoptosis. In addition, activation of the PI3K/Akt signaling pathway in proliferation and apoptosis regulatory signal pathways was confirmed, which is consistent with previous studies. Akt directly regulates endogenous antiapoptotic effectors of the Bcl-2 family members and phosphorylates apoptosis cascade-related regulatory proteins that share the Bcl-2 homogenous domain 3. Bad belongs to this endogenous antiapoptotic Bcl-2 family, and p-Akt directly phosphorylates Bad by combining BH3 with apoptosis cascade-related regulatory proteins to further regulate protein bioactivity, inhibit antiapoptotic effects and induce apoptosis $(19,20)$. Previous studies have indicated that p-Akt directly phosphorylates the prostate apoptosis response protein (Par-4) to inactivate apoptosis induction effects and maintain carcinoma cell survival. Forkhead box (Fox) proteins have conserved Akt phosphorylation sequences. Once Fox proteins are phosphorylated by Akt, they migrate out of the nucleus and chelate with cytoplasmic proteins, losing their facilitating effects on the transcription of apoptosis related genes, Fas-L and Bim, which induce apoptosis, arrest the cell cycle and stimulate metabolism (21). The PI3K/Akt signaling pathway blocks cyclin production or inhibits CDKI activity via multiple signaling pathways (22). p-Akt binds specifically to the p53 negative regulatory protein, MDM2, at Ser166 and Ser186 and relocates MDM2 to the nuclei. MDM2 interacts with p53, inducing its inactivation by blocking the arrest of p53 in cell cycle stage G1, thereby promoting the cell cycle. Akt phosphorylates GSK-3, which is continuously produced in resting cells, and induces the phosphorylation of cyclin D1 while being degraded by the endogenous proteasome. This results in an extended G1 stage, and p-Akt indirectly protects cyclin D1 by inactivating GSK-3. In addition, $\mathrm{p}$-Akt enhances $\beta$-catenin stability, thereby improving the transcription efficiency of the LEF transcription factor, which results in the improved transcription and expression of cyclin D1 (23). To conclude, the present study indicates that the PI3K/Akt signaling pathway regulates the proliferation pathways of ovarian carcinoma cells, improving the proliferation activity and further enhancing invasion and metastasis. However, the PI3K/Akt signaling pathway also regulates the apoptosis-related proteins of carcinoma cells, which enhance the activation of endogenous antiapoptotic effectors and/or inhibit the expression and activation of apoptosis-associated proteases, thereby restraining apoptosis. The PI3K/Akt signaling pathway may play a key regulatory role in the development of OSC and become a primary target for gene therapy. Future in-depth studies may further contribute to the understanding of the mechanisms of ovarian carcinoma development and provide clinical guidance.

\section{Acknowledgements}

The authors thank Yunshui Peng from the Chinese Journal of Anesthesiology for writing assistance and Zhimin Zheng from the First Hospital of Shijiazhuang for support.

\section{References}

1. Mooney SJ, Winner M, Hershman DL, et al: Bowel obstruction in elderly ovarian cancer patients: a population-based study. Gynecol Oncol 129: 107-112, 2013.

2. Oberaigner W, Minicozzi P, Bielska-Lasota M, et al: Survival for ovarian cancer in Europe: the across-country variation did not shrink in the past decade. Acta Oncol 51: 441-453, 2012.

3. Saldanha SN and Tollefsbol TO: Pathway modulations and epigenetic alterations in ovarian tumorbiogenesis. J Cell Physiol: Sep 16, 2013 (Epub ahead of print).

4. Touma R, Kartarius S, Harlozinska A, Götz C and Montenarh M Growth inhibition and apoptosis induction in ovarian cancer cells. Int J Oncol 29: 481-488, 2006.

5. Staal SP: Molecular cloning of the akt oncogene and its human homologues AKT1 and AKT2: amplification of AKT1 in a primary human gastric adenocarcinoma. Proc Natl Acad Sci USA 84: 5034-5037, 1987.

6. Bellacosa A, Kumar CC, Di Cristofano A and Testa JR: Activation of AKT kinases in cancer: implications for therapeutic targeting. Adv Cancer Res 94: 29-86, 2005.

7. Wang Y, Helland A, Holm R, Kristensen GB and Børresen-Dale AL: PIK3CA mutations in advanced ovarian carcinomas. Hum Mutat 25: 322, 2005.

8. Comstock CE, Revelo MP, Buncher CR and Knudsen KE: Impact of differential cyclin D1 expression and localisation in prostate cancer. Br J Cancer 96: 970-979, 2007.

9. Liao DJ, Thakur A, Wu J, Biliran H and Sarkar FH: Perspectives on c-Myc, Cyclin D1, and their interaction in cancer formation, progression and response to chemotherapy. Crit Rev Oncog 13: 93-158, 2007.

10. Meng Q, Xia C, Fang J, Rojanasakul Y and Jiang BH: Role of $\mathrm{PI} 3 \mathrm{~K}$ and AKT specific isoforms in ovarian cancer cell migration, invasion and proliferation through the p70S6K1 pathway. Cell Signal 18: 2262-2271, 2006.

11. Datta SR, Dudek H, Tao X, et al: Akt phosphorylation of BAD couples survival signals to the cell-intrinsic death machinery. Cell 91: 231-241, 1997.

12. Kane LP, Shapiro VS, Stokoe D and Weiss A: Induction of NF-kappaB by the Akt/PKB kinase. Curr Biol 9: 601-604, 1999.

13. Fang X, Yu SX, Lu Y, et al: Phosphorylation and inactivation of glycogen synthase kinase 3 by protein kinase A. Proc Natl Acad Sci USA 97: 11960-11965, 2000.

14. Philp AJ, Campbell IG, Leet C, et al: The phosphatidylinositol 3'-kinase p85alpha gene is an oncogene in human ovarian and colon tumors. Cancer Res 61: 7426-7429, 2001.

15. Noske A, Kaszubiak A, Weichert W, et al: Specific inhibition of AKT2 by RNA interference results in reduction of ovarian cancer cell proliferation: increased expression of AKT in advanced ovarian cancer. Cancer Lett 246: 190-200, 2007.

16. Malumbres $M$ and Barbacid M: Cell cycle, CDKs and cancer: a changing paradigm. Nat Rev Cancer 9: 153-166, 2009.

17. Sherr CJ: Mammalian G1 cyclins. Cell 73: 1059-1065, 1993.

18. Lee SH, Lee JK, Jin SM, et al: Expression of cell-cycle regulators (cyclin D1, cyclin E, p27kip1, p57kip2) in papillary thyroid carcinoma. Otolaryngol Head Neck Surg 142: 332-337, 2010.

19. Maurer U, Charvet C, Wagman AS, Dejardin E and Green DR: Glycogen synthase kinase-3 regulates mitochondrial outer membrane permeabilization and apoptosis by destabilization of MCL-1. Mol Cell 21: 749-760, 2006.

20. Robey RB and Hay N: Mitochondrial hexokinases, novel mediators of the antiapoptotic effects of growth factors and Akt. Oncogene 25: 4683-4696, 2006.

21. Dummler B and Hemmings BA: Physiological roles of PKB/Akt isoforms in development and disease. Biochem Soc Trans 35: 231-235, 2007.

22. Manning BD and Cantley LC: AKT/PKB signaling: navigating downstream. Cell 129: 1261-1274, 2007.

23. Dey A, Tergaonkar V and Lane DP: Double-edged swords as cancer therapeutics: simultaneously targeting p53 and NF-kappaB pathways. Nat Rev Drug Discov 7: 1031-1040, 2008. 